\title{
Diagnostic Utility of Bronchoalveolar Lavage and Bronchial Brush Cytology in Lung Lesions
}

\author{
Jofy George* and Umashankar T \\ Department of Pathology, Father Muller Medical College and Hospital, Mangalore, Karnataka, India
}

\begin{abstract}
Background: In 1960's, flexible fiberoptic bronchoscope, was first used to give a better yield to diagnose pulmonary diseases. Bronchial brushing cytology (BBC) and Broncho-alveolar lavage (BAL) are two important adjuncts done along with bronchoscopic biopsy. Bronchial brushings yield better diagnostic material than simple exfoliative cytology. Therefore, both techniques are of much diagnostic value and is widely accepted as it is safe, economical and evaluation requires much less time.
\end{abstract}

Methods: All the bronchial brush and bronchoalveolar lavage smears obtained from January 2018 to December 2018 were assessed. Correlation was done with histopathology wherever was available.

Result: BBC showed a sensitivity and specificity of $59.28 \%$ and $100 \%$. The positive predictive value and negative predictive value were $100 \%$ and $47.62 \%$. BAL showed a sensitivity and specificity of $51 \%$ and $100 \%$. The positive predictive value and negative predictive value was $100 \%$ and $43.18 \%$. The diagnostic accuracy of BBC and BAL were $70.27 \%$ and $64.86 \%$.

Conclusion: Universally bronchial washings and bronchial brushings should be considered important adjunct to bronchoscopic biopsies to diagnose pulmonary lesions as both are safe, economical and can will give better diagnostic yield if incorporated together.

\section{Keywords: Bronchoalveolar Lavage, Bronchial Brush Cytology, Bronchogenic Carcinoma,} Adenocarcinoma, Squamous Cell Carcinoma, Bronchial Washings.

\section{Introduction}

Exfoliative cytology, to study the cells of respiratory tract was first used in 1845. It was in 1919, the ability to diagnose pulmonary diseases was first appreciated. However, it was not until 1950's, that pulmonary cytology came into. ${ }^{[1]}$ In 1960's when flexible fiber-optic bronchoscope was developed, it improved the technique for to give a better yield to diagnose pulmonary diseases. ${ }^{[2]}$

Different diagnostic modalities are available for early diagnosis for lung lesions which include; bronchoscopy, bronchial biopsy, brushing, washing cytology. Combined use of these modalities yields the best results.

Bronchoalveolar lavage (BAL) is the saline lavage of lower respiratory tract. A larger area of alveolar compartment is explored and provides cellular as well as non-cellular constituents from the same. BAL is one of the initial procedures in diagnosis of interstitial lung diseases. It is also useful in elucidation of pulmonary infiltrates and identification of opportunistic infections. ${ }^{[1][2][3]}$

Bronchial brushing cytology (BBC) yields better diagnostic material than simple exfoliative cytology and results in wider sampling than biopsies. ${ }^{[1][3]}$ The cells obtained are directly derived from the tissue and are better preserved. ${ }^{[3]}$ Therefore, cells obtained by BBC do not show changes due to degeneration or necrosis. If inflammatory cells are noted, then it is derived from the lesion itself, not the result of secondary inflammatory event. ${ }^{[3]}$

Both procedures are safe, economical and evaluation requires much less time. However, there is still disagreement as to the value and reliability of BAL and $\mathrm{BBC}$ in comparison with histology for the diagnosis of pulmonary lesions, especially malignancy. This study was undertaken in the Department of Pathology, Fr Muller Medical College, to make an attempt to study the diagnostic utility of BAL and $\mathrm{BBC}$ in pulmonary lesions (both neoplastic and non-neoplastic). The objectives of the study were

- To study the cytopathological features of lung lesions in BAL.

- To study the cytopathological features of lung lesions in BBC.

- To compare cytopathological findings of BAL and BBC.

- To correlate cytopathological findings of BAL and $\mathrm{BBC}$ with histopathology of these lesions wherever possible.

The introduction of fiber-optic bronchoscopy revolutionized the cytologic sampling of lung lesions. It contributed to 
better assessment of pulmonary lesions that were either not detectable by roentgenologic examination or unsuspected. ${ }^{[3]}$

Raiza D et al, in their study observed that, the BAL showed sensitivity of $80.5 \%$, specificity of $92.85 \%$ and accuracy of $80.5 \%$. The male to female ratio was $6: 1$ and mean age of presentation was 45 to $60 y$ rs in both males and females. Complete cytological and histological correlation was observed in $80.5 \%$ cases. ${ }^{[4]}$

In a study done by Behura et al, out of the 43 BBC cases, $88 \%$ were males. Most of the cases were inflammatory and predominantly in the $5^{\text {th }}$ to $6^{\text {th }}$ decade. Out of 43 cases, 27 cases $(62.79 \%)$ were of inflammatory origin and 13 $(30.23 \%)$ malignant cases. Age range of inflammatory cases was from 21-70 years and malignant cases were from 31-70 years. ${ }^{[5]}$

Dhawan $\mathrm{S}$ et al showed that squamous cell carcinoma was the most common malignancy, followed by small cell carcinoma. The accuracy of Bronchial Brush Cytology in the diagnosis of Squamous Cell Carcinoma was $81.57 \%$; in Bronchogenic Adenocarcinoma accuracy was $80 \%$, in Small Cell Anaplastic Carcinoma $83.33 \%$ and in Large Cell Carcinoma it was $100 \%$. ${ }^{[6]}$

Rao S et al revealed that, BAL has low sensitivity and high specificity and is a valuable method that provides significant information in the evaluation of lung pathology. BAL showed a sensitivity of $52.63 \%$ and specificity of $80 \%$ with accuracy of $62.06 \%$. ${ }^{[7]}$

In a study done by R. Giti et al, where they studied the efficacy of bronchial washing and bronchial brush cytology in diagnosing non-neoplastic lesions, found that male to female ratio was 6:1. Most common non-neoplastic lesion was tuberculosis $(23 \%)$ followed by pneumonia $(19 \%)$. They also revealed that $31 \%$ were false negative for lung cancer. ${ }^{[8]}$

In a study done by Prakash et al, it was found that, Sensitivity of BAL and BB was found to be 47.61 and $65.07 \%$ respectively, whereas specificity of BAL and $\mathrm{BB}$ was 75 and $75 \%$ respectively. Accuracy of BAL was $44.77 \%$, BB was $65.67 \%$, Bronchial brushing alone diagnosed 80 cases, out of 100 malignant cases. ${ }^{[9]}$

In another study done by Tomar et al, while comparing between Bronchoalveolar Lavage, Bronchial Brush Cytology and Fine Needle Aspiration Cytology; sensitivity of BBC was found to be $65.07 \%$ and specificity was $75 \%$. ${ }^{[10]}$

\section{Materials and Methods}

This study was conducted in Father Muller Medical College Hospital (FMMCH), Mangalore, Karnataka, India for the time period of 12 months, from $1^{\text {st }}$ Jan 2018 to $31^{\text {st }}$
December 2018 as an observational comparative study. It was a time bound study with a minimum sample size 66 cases.

All the bronchial brush smears and Broncho-alveolar lavage samples submitted to the Department of Pathology, FMMCH, Mangalore for cytopathological study during the period from January 2018 to December 2018 were included in the study. However, cases were excluded if any of the two samples were not received. The clinical data was obtained from clinical records. The histopathology of the same was obtained, if it was submitted for evaluation in the histopathology section of the pathology department.

For BBC, a brush was applied to the surface of an endobronchial lesion under the bronchoscopy guidance with prior consent and the cells which are entrapped were smeared onto a glass slide.

The BAL material was obtained from the bronchial tree by instilling isotonic saline and re-aspirating it. Samples were prepared into air-dried and wet-fixed smears. These slides were then stained with Papanicolaou and May Grunwald Giemsa stain.

\section{Results}

In this study, a total of 70 cases, were studied in the time frame of one year, Jan 2018 to December 2018. Out of the 70 cases, BBC diagnosed 54 cases $(77.1 \%)$ as reactive and rest $(22.8 \%)$ where suspicious and positive for malignancy. Through BAL, 56 cases $(80 \%)$ were diagnosed as reactive and 14 cases $(20 \%)$.

The most common age group of presentation was age range of 51-60 years with 27 cases (38.5\%). Following table shows distribution of cases according to age. The distribution of cases according to the gender showed a male predominant population in 70 cases that we studied. The male to female ratio was 3.3:1. Out of the 70 cases, 37 cases had histopathological correlation. Each of the 37 cases had BBC, BAL and histopathology findings.

In $\mathrm{BBC}$, the 21 cases were diagnosed as reactive (RCT) and 10 had correlation with histopathology. However, rest 11 turned out to be positive for malignancy in histopathology. 16 cases diagnosed as positive (POS) and suspicious (SUS) for malignancy in $\mathrm{BBC}$, of which 10 were subtyped and all were correlating with histopathology.

In BAL, 24 cases were diagnosed as reactive or benign $(\mathrm{BN})$, out of which 10 turned out to be reactive itself in histopathology and 13 were positive and suspicious for malignancy in histopathology. All the 13 cases were subtyped and had a correlation with histopathology. 14 cases were positive in BAL and was $100 \%$ correlating. 
When we compared the results of $\mathrm{BBC}$ and BAL, 54 cases were reactive in $\mathrm{BBC}$, out of which 52 were reactive in BAL and 2 turned out to be suspicious /positive for malignancy. 16 cases were positive in $\mathrm{BBC}$, out of which 12 were positive in BAL also. Only 4 was reactive /benign in BAL. Table 2 shows comparative analysis of cases according distribution in BBC and BAL.

Out of the 37 cases which had histopathology, 10 were reactive and 27 were suspicious / positive for malignancy. 10 cases which were reactive in histopathology were correctly reported in BBC and BAL.

27 cases which were suspicious/positive for malignancy in HPE, out of which 14 were positive BAL, of which 2 were

Table 1: Distribution of cases according to age.

\begin{tabular}{|c|c|}
\hline Age range (years) & Number of cases (n) (\%) \\
\hline $21-30$ & $2(2.85 \%)$ \\
\hline $31-40$ & $3(4.2 \%)$ \\
\hline $41-50$ & $10(14.2 \%)$ \\
\hline $51-60$ & $27(38.5 \%)$ \\
\hline $61-70$ & $20(28.5 \%)$ \\
\hline $71-80$ & $7(10 \%)$ \\
\hline$>80$ & $1(1.4 \%)$ \\
\hline
\end{tabular}

Table 2: BBC vs BAL.

\begin{tabular}{|c|c|c|}
\hline \multicolumn{2}{|c|}{ BBC/BAL } \\
\hline BBC & \multicolumn{2}{|c|}{ BAL } \\
\hline REACTIVE & BN/RCT & 2 \\
\hline SUS/POS & 52 & 12 \\
\hline TOTAL & 4 & 14 \\
\hline
\end{tabular}

Table 3: HPE vs BBC and BAL.

\begin{tabular}{|c|c|c|c|c|c|c|c|c|}
\hline \multicolumn{9}{|l|}{ HPE } \\
\hline & \multicolumn{4}{|c|}{ BAL } & \multicolumn{4}{|c|}{ BBC } \\
\hline \multirow{3}{*}{$\begin{array}{c}\text { REACTIVE } \\
(10)\end{array}$} & \multicolumn{2}{|c|}{ REACTIVE } & \multicolumn{2}{|c|}{ SUS/POS } & \multicolumn{2}{|c|}{ REACTIVE } & \multicolumn{2}{|c|}{ SUS/NEO } \\
\hline & \multicolumn{2}{|c|}{$\mathrm{BBC}$} & \multicolumn{2}{|c|}{ BBC } & \multicolumn{2}{|c|}{$\mathrm{BAL}$} & \multicolumn{2}{|c|}{ BAL } \\
\hline & REACTIVE & SUSP/NEO & REACTIVE & SUSP/POS & REACTIVE & SUSP/POS & REACTIVE & SUSP/POS \\
\hline TOTAL & 10 & 0 & 0 & 0 & 10 & 0 & 0 & 0 \\
\hline \multirow{4}{*}{$\begin{array}{c}\text { SUS/POS } \\
(27)\end{array}$} & \multicolumn{4}{|c|}{ BAL } & \multicolumn{4}{|c|}{ BBC } \\
\hline & \multicolumn{2}{|c|}{ REACTIVE } & \multicolumn{2}{|c|}{ SUS/POS } & \multicolumn{2}{|c|}{ REACTIVE } & \multicolumn{2}{|c|}{ SUS /POS } \\
\hline & \multicolumn{2}{|c|}{ BBC } & \multicolumn{2}{|c|}{ BBC } & \multicolumn{2}{|c|}{$\mathrm{BAL}$} & \multicolumn{2}{|c|}{$\mathrm{BAL}$} \\
\hline & REACTIVE & SUSP/POS & REACTIVE & SUSP/POS & REACTIVE & SUSP/POS & REACTIVE & SUSP/POS \\
\hline TOTAL & 9 & 4 & 2 & 12 & 9 & 2 & 4 & 12 \\
\hline
\end{tabular}

reactive and 12 were positive in $\mathrm{BBC}$. 13 were reactive in BAL, of which 9 were reactive and 4 were positive in BBC. Of the 27 positive cases in histopathology, BBC was reactive for 11 cases, of which 9 were reactive and 2 were suspicious /positive in BAL. 16 were positive in BBC, of which 12 were positive and 4 were reactive in BAL.

BBC showed a sensitivity and specificity of $59.28 \%$ and $100 \%$. The positive predictive value and negative predictive value were $100 \%$ and $47.62 \%$. BAL showed a sensitivity and specificity of $51 \%$ and $100 \%$. The positive predictive value and negative predictive value was $100 \%$ and $43.18 \%$. The diagnostic accuracy of BBC and BAL were $70.27 \%$ and $64.86 \%$. 


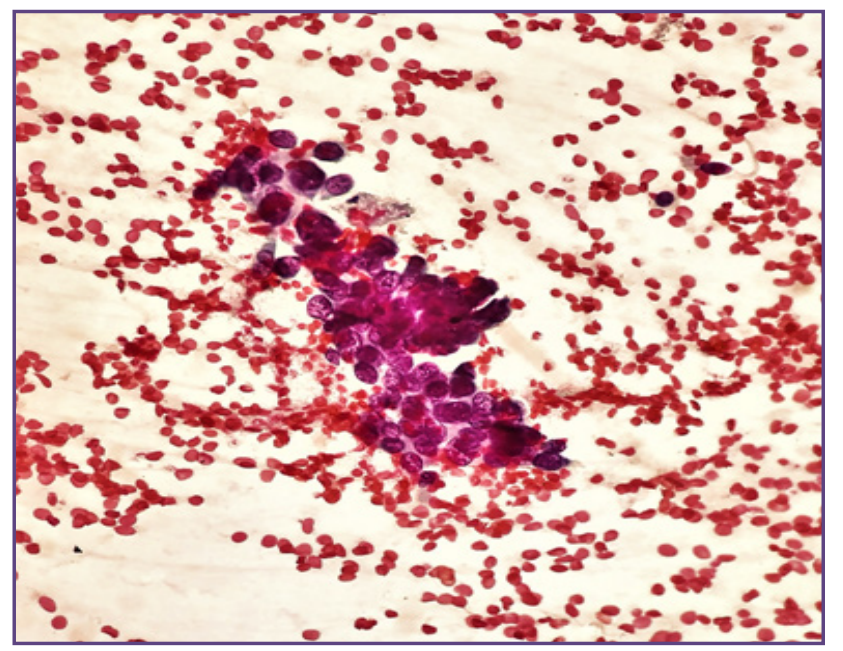

Fig. 1: BBC - Adenocarcinoma, Pap stain-40x.

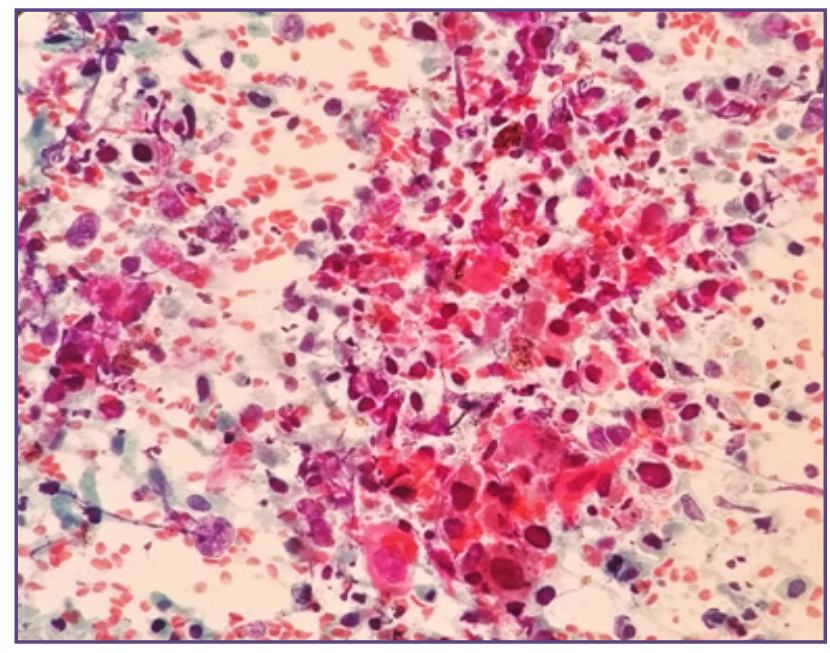

Fig. 3: BBC, Squamous cell carcinoma (Pap stain -40 x).

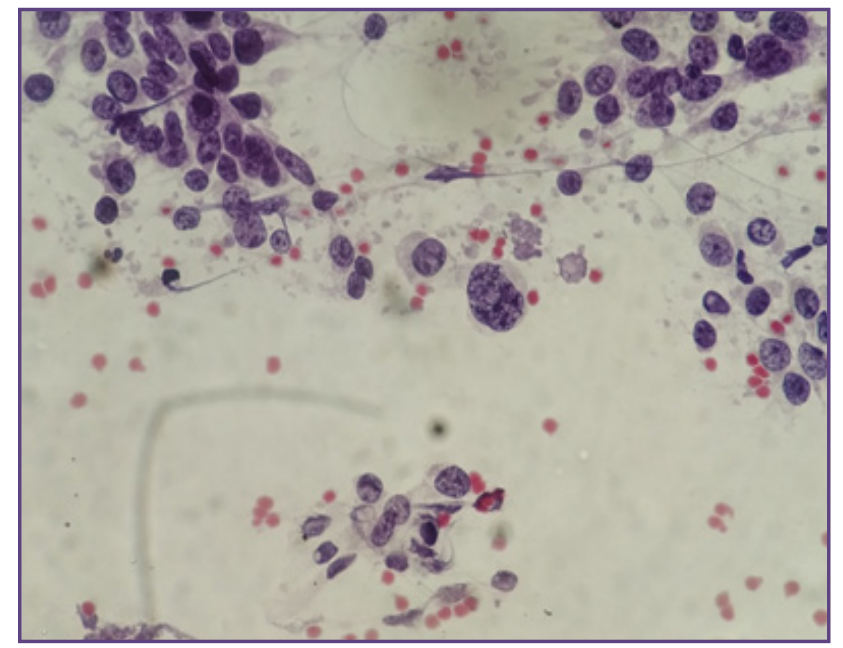

Fig. 2: BAL -Adenocarcinoma (Pap stain-40x).

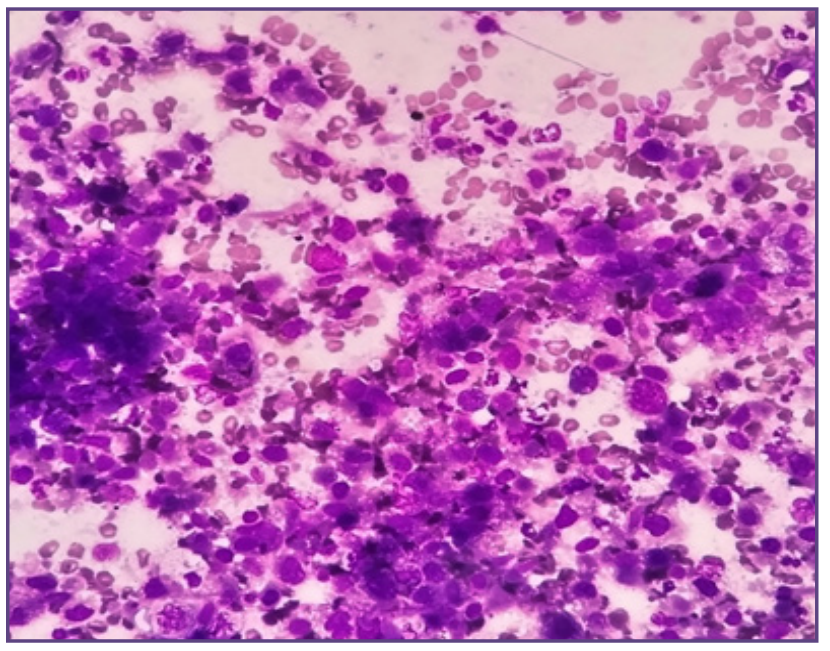

Fig. 4: BBC, Squamous cell carcinoma (MGG stain-40x).

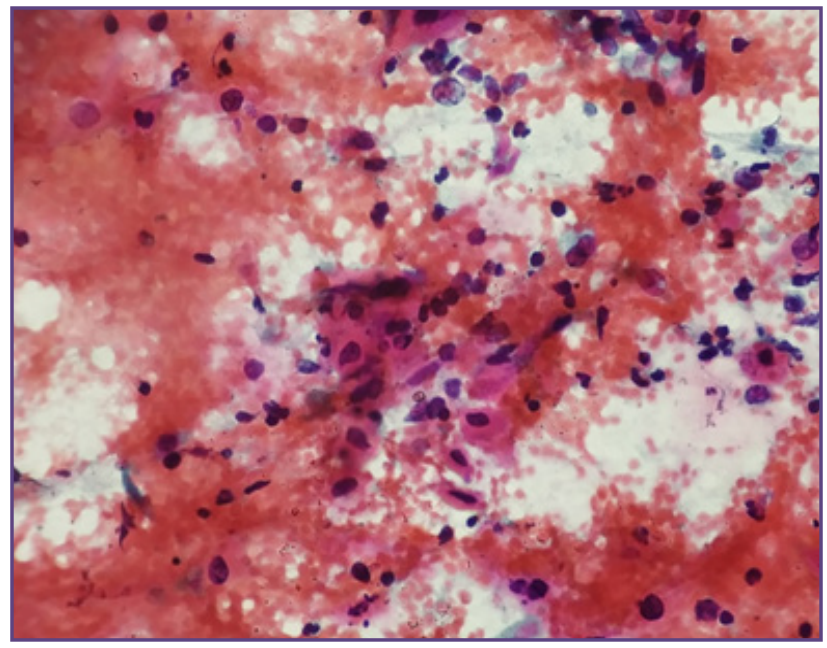

Fig. 5 : BAL, Squamous cell carcinoma (Pap stain-40x). 


\section{Discussion}

We studied 70 cases, in a time period of one year which had both BBC and BAL samples sent for a patient. As BBC was introduced in our institute only this year, we wanted to study the diagnostic accuracy of the same. The mean age group of our study was between 51-60 years. Dhawan et al showed that the mean age group was $61-70$ years. ${ }^{[6]}$ Giti et al showed similar results where the mean age group was 57 years. ${ }^{[8]}$

The male to female ratio in our study was $3.3: 1$. The male population was seen to be more than the female population. This was similar to the other studies done by Raiza D et al, and Giti et al where the male to female ratio was $6: 1 .{ }^{[4,8]}$ Behura et al also showed $88 \%$ males being affected. ${ }^{[5]}$

In our study, 70 cases were been studied and out of that 54 cases $(77.1 \%)$ were reactive and rest $(22.8 \%)$ where suspicious and positive for malignancy. Similar results were seen in other studies which showed predominant lesions being the inflammatory etiology showing reactive bronchial cells. ${ }^{[4-10]}$

The sensitivity and specificity of BAL and BBC in our study was 51\%, 100\% 59.28\% and 100\% respectively. Similar results were observed by Prakash et al, and found that, sensitivity of BAL and BBC was found to be 47.61 $\%$ and $65.07 \%$ respectively, whereas specificity of BAL and $\mathrm{BBC}$ was $75 \%$ each respectively. ${ }^{[9]}$

Two cases were reported as reactive in $\mathrm{BBC}$, and had turned out to be neoplastic in BAL. One was diagnosed as squamous cell carcinoma and other was reported as adenocarcinoma in histopathology. In these two cases, the brush probably could not reach the lesion and hence was not able to collect the tumour cells. However, BAL, as tumour cells shed in carcinomas, it would be easy to collect such cells and can be appreciated in the microscopy.

One case was correctly diagnosed as neoplastic in BBC was reactive in BAL. As BBC takes samples directly from the lesion, the cellular details are preserved very well and hence for this case it was able to give a correct diagnosis. In histopathology it was reported as poorly differentiated carcinoma. In BAL, the amount of reactive bronchial epithelial cells would be more and preservation of cellular material is not good as BBC. Hence, it is difficult to differentiate reactive cells and tumour cells.

3 cases were reported suspicious in $\mathrm{BBC}$ and was reactive in BAL. among the three cases, one was small cell carcinoma, other poorly differentiated carcinoma and third was squamous cell carcinoma. Similar difficulties were observed in the other studies.

\section{Conclusion}

Universally $\mathrm{BAL}$ and $\mathrm{BBC}$ are considered important adjunct to bronchoscopic biopsies to diagnose pulmonary lesions. As both techniques are safe, economical, feasible and have a good diagnostic accuracy it can be concluded both should be incorporated in diagnosing a lung lesion to get a higher yield.

\section{Acknowledgements}

I would like to thank the faculty, my colleagues, and technical staff of the Department of Pathology, Father Muller Medical College, Mangalore, Karnataka, India for helping and guiding me through this work.

\section{Funding}

None

\section{Competing Interests}

None declared

\section{Reference}

1. Koss LG, Melamed MR, editors. Koss' diagnostic cytology and its histopathologic bases. Lippincott Williams \& Wilkins; 2006.

2. Kocjan G, Gray W, Vielh P, Levine T, Kardum-Skelin I. Diagnostic Cytopathology Essentials E-Book. Elsevier Health Sciences; 2013 May 8.

3. Cibas ES, Ducatman BS. Cytology E-Book: diagnostic principles and clinical correlates. Elsevier Health Sciences; 2013.

4. Raiza D, Rout S, Reddy KP, Ramalaxmi PV, Prithvi BK, Harikishan KS. Efficacy of bronchial wash and brush cytology and its correlation with biopsy in lung lesions. Int $\mathbf{J}$ Health Res Mod Integr Health Sci. 2014;3:21-4.

5. Behura A, Rao KM. BRONCHOSCOPIC BRUSH CYTOLOGY IN THE DIAGNOSIS OF LUNG LESIONS. group. 2018 Jan 1;21(30):9-34.

6. Dawan S. Efficacy of Bronchial Brushing Cytology and Its Correlation with Biopsy in Lung Tumours At tertiary care Hospital northern western. Journal of Medical science and clinical research. 2017;5(7).

7. Rao S, Rao S, Lal A, Barathi G, Dhanasekar T, Duvuru P. Bronchial wash cytology: A study on morphology and morphometry. Journal of Cytology. 2014;31(2):63.

8. Giti R, Hosseinzadeh M. Efficacy of Bronchial Washing and Brushing Cytology in the Diagnosis of Non-Neoplastic Lung Diseases. Acta Medica Iranica. 2017 Dec 2:636-41.

9. Prakash P, Agarwal P, Dasgupta A, Gahoi K. Study of Diagnostic Efficacy of Bronchoalveolar Lavage and 
Bronchoscopic Brush Cytology in Clinico-radiologically Suspected Cases of Lung Malignancies.2018 Jan;19(1):22.

10. Tomar V, Vijay N, Nuwal P, Dixit R. Comparative study of bronchoalveolar lavage, bronchial brushing, and FNAC in diagnosing malignant neoplasms of lungs. Journal of cytology. 2016 Oct;33(4):210

\section{*Corresponding author:}

Dr. Jofy George, Post Graduate (Resident), Department of Pathology, Father Muller Medical College and Hospital, Mangalore, Karnataka, India

Email: jofygeorge0404@gmail.com

Date of Submission : 07/11/2019

Date of Acceptance : 26/07/2020

Financial or other Competing Interests: None.

Date of Publication : 30/08/2020 